\title{
Nefrectomía simple por puerto único (LESS) asistida por robot (da Vinci)*
}

\author{
Drs. OCTAVIO A. CASTILLO C. ${ }^{1,2,3}$, IVAR VIDAL M. ${ }^{1}$, FRANCISCO SEPÚLVEDA T. ${ }^{1}$ \\ 1 Unidad de Urología y Centro de Cirugía Robótica, Clínica Indisa. \\ 2 Facultad de Medicina, Universidad Andrés Bello. \\ 3 Facultad de Medicina, Universidad de Chile. \\ Santiago, Chile.
}

\begin{abstract}
\section{Single-site port robotic-assisted simple nephrectomy (LESS)}

Introduction: Minimally invasive surgery in urology is rapidly advancing and Laparo-endoscopic single-site surgery (LESS) is not the exception. Such laparoscopic procedures are technically challenging and require an experienced laparoscopic surgeon due to the lack of port placement triangulation and instrument clashing. The benefit of the da Vinci surgical system has recently introduced to LESS. We present two cases of robotic LESS nephrectomy. Matherials and Methods: Two patients, a female of 23 years old, diagnosed with right renal atrophy secondary to chronic pyelonephritis and one male patient with diagnosis of left staghorn calculi and renal atrophy. Both underwent to a total nephrectomy assisted by the da Vinci S surgical system through a single port incision using the GelPoint ${ }^{\circledR}$ access system. Results: The first surgery was performed without incidents or conversion. The second patient required the installation of an additional robotic port for triangulation. The dock time and the mean operative time was 18 and $110 \mathrm{~min}$. The mean estimated blood loss was 100 cc and the hospital stay was 27 hours. There were no complications. Conclusions: LESS robotic surgery is feasible using current robotic systems. However, there are several limitations. The design of specific technology for the use of the robot through single incision can solve this problem.
\end{abstract}

Key words: Laparoscopic surgery, nephrectomy, robotic surgery, LESS.

\section{Resumen}

Introducción: La cirugía mínimamente invasiva en urología avanza rápidamente y la cirugía laparoendoscópica a través de puerto único (LESS) no es la excepción. Esta técnica por vía laparoscópica presenta mucha dificultad y requiere de un cirujano laparoscópico experimentado debido a la falta de triangulación y el cruce de los instrumentos. Los beneficios del sistema quirúrgico da Vinci® han sido introducidos recientemente en LESS. Presentamos dos casos de nefrectomía LESS asistida por robot. Materiales y Métodos: Dos pacientes, la primera de sexo femenino y 23 años de edad, con diagnóstico de atrofia renal secundaria a pielonefritis crónica derecha y un segundo paciente de sexo masculino con diagnóstico de litiasis coraliforme izquierda y atrofia renal. Ambos pacientes fueron sometidos a una nefrectomía total asistida por el sistema quirúrgico robótico da Vinci $S$ a través de un puerto único utilizando el dispositivo de acceso GelPoint ${ }^{\circledR}$. Resultados: La primera cirugía fue llevada a cabo sin incidentes, sin necesidad de conversión. En el segundo

*Recibido el 17 de agosto de 2010 y aceptado para publicación el 2 de diciembre de 2010.

Correspondencia: Dr. Octavio A. Castillo C.

Apoquindo 3990, Oficina 809, C.P.: 755 0112, Santiago, Chile. Fax: (56-2) 2282524

octavio.castillo@indisa.cl 
paciente fue necesaria la instalación de un puerto robótico adicional para triangulación. El tiempo de acoplamiento y operatorio promedio fue de 18 y 110 min. El sangrado promedio estimado fue de 100 cc y la estadía hospitalaria promedio de 27 horas. No hubo complicaciones. Conclusiones: La cirugía robótica LESS es posible de realizar utilizando los sistemas robóticos actuales. Sin embargo, existen diversas limitaciones. El diseño de tecnología específica para el uso del robot por puerto único podrá solucionar este problema.

Palabras clave: Cirugía laparoscópica, nefrectomía, cirugía robótica, LESS.

\section{Introducción}

La cirugía laparoscópica ha experimentado diversos cambios desde su introducción en nuestro país ${ }^{1}$. El deseo de minimizar el malestar postoperatorio del paciente y mejorar la cosmética a estimulado el interés por la cirugía a través de orificios naturales (NOTES) y la cirugía laparo-endoscópica a través de puerto único (LESS). Se han reportado más de 100 procedimientos urológicos LESS con buenos resultados ${ }^{2}$. A pesar de que los resultados son prometedores, esto no debe anteponerse a las dificultades técnicas asociadas a la cirugía LESS. Este tipo de cirugía presenta un gran desafío para el cirujano principalmente debido a la falta de triangulación y la colisión de los instrumentos laparoscópicos estándar ${ }^{3}$, presentando la sutura intracorpórea una dificultad adicional. La aplicación del sistema quirúrgico da Vinci (Intuitive Surgical, Inc., Sunny-Valley, California, USA) permite mayor maniobrabilidad quirúrgica y mejora la ergonomía durante LESS. Nosotros presentamos 2 casos de nefrectomía robótica LESS, que son la primera comunicación en nuestro país y en Latinoamérica.

\section{Material y Método}

El primer caso es una paciente de sexo femenino, de 23 años de edad con diagnóstico de pielonefritis recurrente derecha. Una cintigrafía renal demostró una función renal izquierda de 11,7\% y una atrofia severa del parénquima renal a derecha. Se decidió una nefrectomía simple y se planeó realizarla por vía laparoscópica asistida por robot a través de un puerto único. El procedimiento fue explicado plenamente al paciente y su familia, los cuales entregaron su consentimiento.

El segundo paciente de sexo masculino, de 46 años de edad, tiene una historia de urolitiasis a repe- tición. Luego de 2 años sin episodios sintomáticos, en chequeo rutinario se pesquiza litiasis coraliforme izquierda completa, asociada a atrofia del parénquima renal, con exclusión renal cintigráfica. Se planificó para una nefrectomía robótica por puerto único.

\section{Descripción de la técnica quirúrgica}

Ambos pacientes fueron colocados en posición de lumbotomía bajo anestesia general. Se realizó una incisión transumbilical en forma de omega de 2,5 cm y $6 \mathrm{~cm}$ respectivamente. Esta incisión se adecua según el tamaño de la pieza a extraer. Luego se procede a instalar el dispositivo de acceso GelPoint (Applied Medical, Rancho Santa Margarita, California, USA), en el cual se pre instalan 2 trócares robóticos de $8 \mathrm{~mm}$ y un trocar de $12 \mathrm{~mm}$ para la cámara (Figura 1), colocando puertos adicionales para el ayudante según necesidad. Se realiza neumoperitoneo hasta $15 \mathrm{mmHg}$. El acoplamiento del sistema quirúrgico daVinci $\mathrm{S}$ se realiza desde la espalda del paciente (Figura 2). La exposición quirúrgica se verificó adecuadamente con el uso del sistema robótico a través de un puerto único y los pasos estándar de la cirugía laparoscópica se replicaron sin inconveniente. En el segundo paciente, debido a un mayor tamaño renal, fue necesaria la instalación de un puerto robótico en hipocondrio izquierdo para una mejor triangulación.

\section{Resultados}

Las características de los pacientes se presentan en la Tabla 1. Destaca la realización de los procedimientos con el sistema quirúrgico da Vinci. Los resultados peri operatorios se demuestran en la Tabla 2. La alimentación se reinició a la mañana siguiente del procedimiento y los 2 pacientes fueron dados de alta antes de 36 hrs.

Tabla 1. Características de los pacientes

\begin{tabular}{|c|c|c|c|c|c|c|}
\hline Paciente & Sexo & Edad & Diagnóstico & Técnica & Tipo de cirugía & Observación \\
\hline 1 & Femenino & 23 & $\begin{array}{l}\text { Pielonefritis } \\
\text { crónica }\end{array}$ & $\begin{array}{l}\text { LESS } \\
\text { robótico }\end{array}$ & Nefrectomía simple & \\
\hline 2 & Masculino & 46 & $\begin{array}{l}\text { Litiasis } \\
\text { coraliforme }\end{array}$ & $\begin{array}{l}\text { LESS } \\
\text { robótico }\end{array}$ & Nefrectomía simple & $\begin{array}{l}\text { Asistencia de puerto robótico en hipo- } \\
\text { condrio izquierdo para triangulación }\end{array}$ \\
\hline
\end{tabular}

*LESS: Laparoendoscopic single site surgery. 


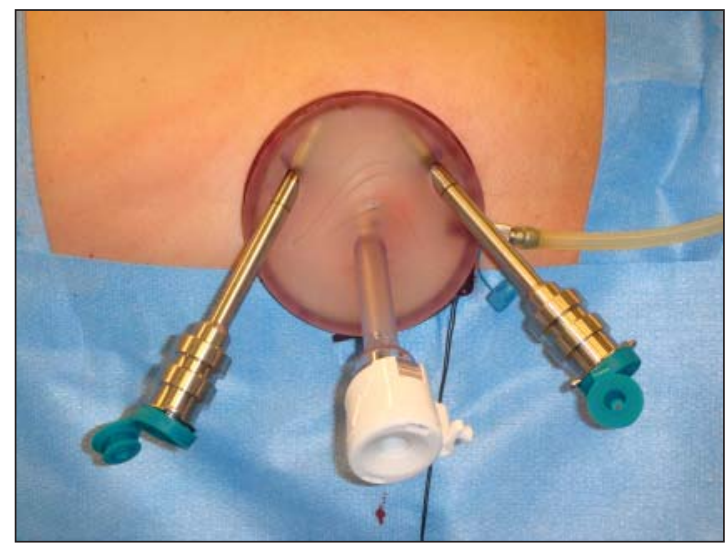

Figura 1. Dispositivo de acceso GelPOINT (Applied Medical, Rancho Santa Margarita, California, USA) con tres trócares.

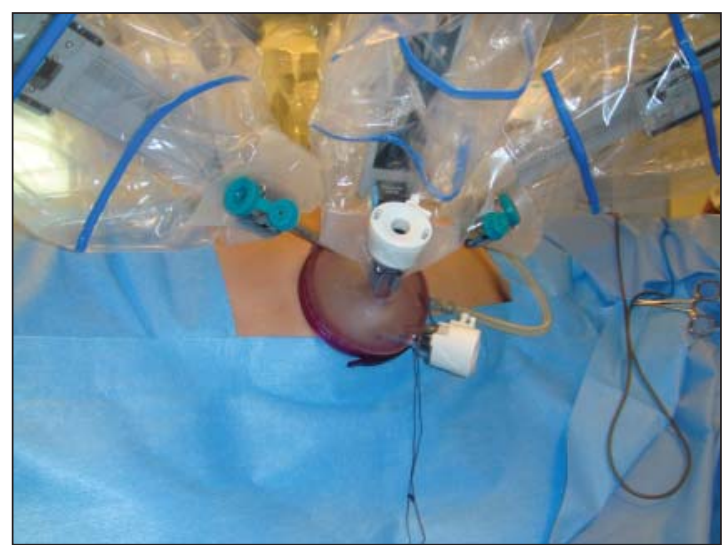

Figura 2. El acoplamiento del sistema quirúrgico DaVinci S se realiza desde la espalda del paciente. 3).

La cicatriz umbilical postoperatoria inmediata es mínima (Figura

El análisis histopatológico final demostró pielonefritis crónica y atrofia renal en ambos casos.

\section{Discusión}

Las ventajas potenciales de la cirugía robótica por puerto único, incluyen una cicatriz pequeña, un acceso mínimamente invasivo,

Tabla 2. Resultados peri operatorios

\begin{tabular}{|ccccc|}
\hline Paciente & $\begin{array}{c}\text { Tiempo } \\
\text { operatorio } \\
\text { (min) }\end{array}$ & $\begin{array}{c}\text { Sangrado } \\
\text { (cc) }\end{array}$ & $\begin{array}{c}\text { Estadía } \\
\text { hospitalaria } \\
\text { (horas) }\end{array}$ & Complicaciones \\
\hline 1 & 90 & 50 & 18 & No \\
2 & 130 & 150 & 36 & No \\
\hline
\end{tabular}

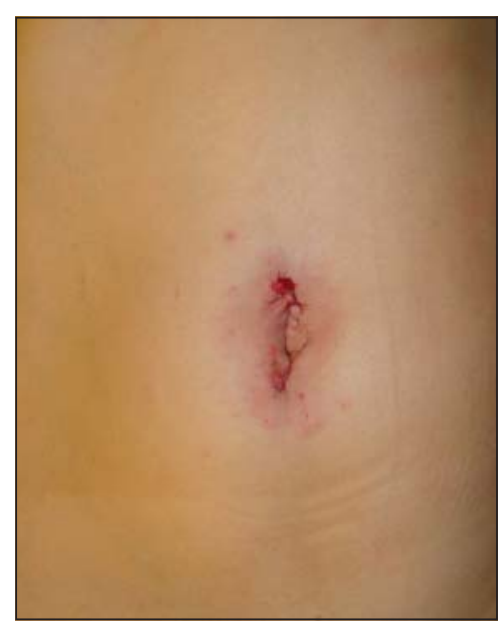

Figura 3. Incisión umbilical de paciente 1.

Tabla 3. Principales experiencias clínicas en LESS robótico

\begin{tabular}{|c|c|c|c|c|c|}
\hline Autor & $\begin{array}{l}\text { Cirugía } \\
\text { realizada }\end{array}$ & Puerto de acceso & $\begin{array}{l}\text { Tiempo opera- } \\
\text { torio (min) }\end{array}$ & $\begin{array}{c}\text { Sangrado } \\
\text { estimado }(\mathrm{ml})\end{array}$ & $\begin{array}{l}\text { Estadía hospi- } \\
\text { talaria (horas) }\end{array}$ \\
\hline Kaouk y cols ${ }^{6}$ & $\begin{array}{l}\mathrm{n}=3 \text {, PR (1), } \\
\text { pieloplastía (1), } \\
\mathrm{NR}(1)\end{array}$ & $\mathrm{R}$-port & $\begin{array}{l}\text { PRL 345, } \\
\text { pieloplastía } \\
\text { 270, NRL } 150\end{array}$ & 250, 80, 200 & ND \\
\hline Kaouk y cols ${ }^{7}$ & $\mathrm{n}=2, \mathrm{NP}$ & Triport & 170 & 100 & 84 \\
\hline Stein y cols ${ }^{8}$ & $\begin{array}{l}\mathrm{n}=4, \\
\text { pieloplastía (2), } \\
\mathrm{NR}(1), \mathrm{NP}(1)\end{array}$ & Gelport & $\begin{array}{l}\text { Pieloplastía } \\
\text { 235, NR 200, } \\
\text { NP } 180\end{array}$ & $37.5,250,600$ & $26,48,48$ \\
\hline Barret y cols ${ }^{9}$ & $\mathrm{n}=1, \mathrm{PR}$ & $\begin{array}{l}3 \text { puertos laparoscópicos, a tra- } \\
\text { vés de incisión umbilical y un } \\
\text { puerto abdominal bajo de } 5 \mathrm{~mm}\end{array}$ & 150 & 500 & No reportado \\
\hline Serie actual & $\begin{array}{l}\mathrm{n}=2 \text {, nefrecto- } \\
\text { mías totales }\end{array}$ & $\begin{array}{l}\text { Gelpoint } 1 \text { caso, Gelpoint }+ \\
\text { puerto de } 8 \mathrm{~mm}\end{array}$ & 90 y 130 & 50 y 150 & 18 y 36 \\
\hline
\end{tabular}

PR: Prostatectomía radical; NR: Nefrectomía radical; NP: Nefrectomía parcial; ND: No disponible. 
menor dolor y resultados comparables a la cirugía abierta. Hasta el momento existe un escaso número y una amplia variedad de procedimientos urológicos LESS con asistencia robótica reportados en la literatura (Tabla 3). Los resultados cosméticos son excelentes y la cicatriz es indetectable cuando esta última se ubica dentro del ombligo. Adicionalmente el dolor post operatorio es mínimo, como ha sido demostrado a través de la escala visual análoga, a pesar de que se necesitan mejores estudios controlados con escalas de dolor validadas para este tipo de cirugía. Es muy precoz para comentar los resultados oncológicos en el desarrollo de esta tecnología, pero los reportes iniciales son prometedores, por lo que se requieren estudios prospectivos y randomizados para comparar los resultados post operatorios entre la cirugía robótica por puerto único y la cirugía laparoscópica tradicional.

Respecto al puerto de acceso, la cirugía robótica a través de puerto único continúa experimentando mejoras. En cirugía NOTES y LESS tradicional, ya hemos utilizado el dispositivo Triport $\AA^{3}$, pero debido a que ésta es una experiencia inicial, decidimos utilizar el nuevo dispositivo conocido como GelPoint (Applied Medical). Este puerto es similar al GelPort, usado para cirugía con asistencia manual, pero más pequeño; no tiene perforaciones pre definidas en la tapa gel, existe un puerto de insuflación a un costado del dispositivo y posee además una sutura atada al extremo que proteje la piel, lo que permite su fácil remoción. El puerto permite que todos los instrumentos sean puestos a través de la tapa gel, aliviando la necesidad de insertar puertos en sitios adyacentes con la consecuente pérdida de pneumoperitoneo. El establecer el puerto de insuflación por un costado, libera espacio en la tapa, lo cual reduce la colisión de los instrumentos. El GelPoint permite además una extracción fácil de los especímenes quirúrgicos durante cirugías resectivas ${ }^{4}$.

A pesar de que la incorporación del sistema da Vinci a la cirugía LESS ha mejorado las limitaciones experimentadas con LESS tradicional, todavía existe una reducción en el rango de movimientos de los instrumentos. Esto nos llevó a la instalación de un puerto robótico adicional en el segundo caso para poder triangular. Esto está en camino de ser resuelto dado que ya existen los prototipos de instrumentos articulados para el sistema da Vinci, lo cual reducirá el movimiento externo de los brazos robóticos y por lo tanto, su colisión.

Todavía estamos lejos de tener el sistema perfecto y la cirugía robótica LESS está todavía en desarrollo. El robot que actualmente está disponible es de gran tamaño y no ha sido diseñado para la cirugía a través de puerto único. El rango limitado de mo- vimiento también es frustrante y se deben realizar mejoras significativas antes que la tecnología se difunda. Esperamos que los avances en el campo de la robótica permitan soslayar estas limitaciones y entregar mejoras en la triangulación, rangos de libertad y visualización ${ }^{5}$.

\section{Conclusión}

El entusiasmo con respecto a la cirugía por puerto único continúa creciendo a un ritmo insospechado. Los avances en instrumentación, acceso y tecnología han reducido las limitaciones iniciales exerimentadas. Con la introducción del sistema robótico da Vinci a la cirugía de puerto único, esperamos disminuir las limitaciones de esta técnica mínimamente invasiva. Si bien se necesita de estudios comparativos prospectivos, los resultados iniciales son prometedores. El desarrollo de un sistema robótico específico para LESS podrá definir el nuevo horizonte de la cirugía a través de puerto único.

\section{Referencias}

1. Castillo O, Sánchez-Salas R, Vidal-Mora I, Albino G, Díaz M, Vitagliano G, y cols. Nefrectomía radical laparoscópica. Nuestra experiencia en 150 pacientes consecutivos. Rev. Chilena de Cirugía 2008;60:297-302.

2. White WM, Haber GP, Goel RK. Single-port urological surgery: single-center experience with the fisrt 200 cases. Urology 2009;74:801-4.

3. Castillo O, Sánchez-Salas R, Vidal-Mora I, Campos R, Ahualli J, Fonerón A et al. Transumbilical laparoscopic simple nephrectomy using a flexible cystoscope and standard laparoscopic instruments. Arch Esp Urol. 2009;62:296-300.

4. White MA, Haber GP, Kaouk JH. Robotic single-site surgery. Curr Opin Urol. 2010;20:86-91.

5. Canes D, Lehman AC, Farritor SM. The future of NOTES instrumentation: flexible robotics and in vivo minirobots. J Endourol. 2009;23:787-92.

6. Kaouk JH, Goel RK, Haber GP. Robotic single-port transumbilical surgery in humans: inicial report. BJU Int. 2009;103:366-9.

7. Kaouk JH, Goel RK. Single-port laparoscopic and robotic partial nephrectomy. Eur Urol. 2009;55:1163-9.

8. Stein RJ, White WM, Goel RK. Robotic laparoendoscopic single-site surgery using gelport as the access platform. Eur Urol. 2010;57:132-6.

9. Barret E, Sánchez-Salas R, Kasraeian A. A transition to laparoendoscopic single-site surgery (LESS) radical prostatectomy: human cadaver experimental and initial clinical experience. J Endourol. 2009;23:135-40. 